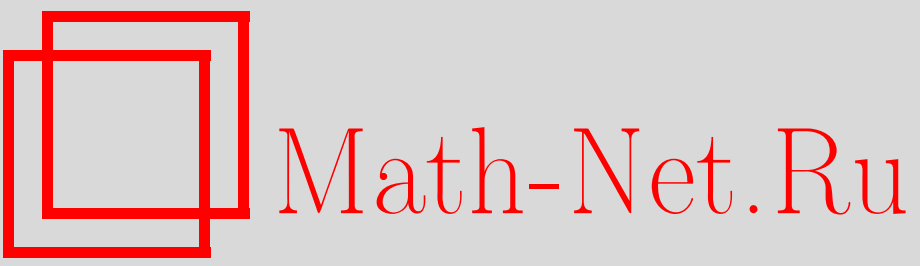

М. Б. Лагутин, Одна модель проникновения газа в пористую среду, Теория вероятн. и ее примен., 2012, том 57, выпуск 2, 377-381

DOI: https://doi.org/10.4213/tvp4453

Использование Общероссийского математического портала Math-Net.Ru подразумевает, что вы прочитали и согласны с пользовательским соглашением

http://www.mathnet.ru/rus/agreement

Параметры загрузки:

IP : 3.93.64.190

26 апреля 2023 г., 15:21:55 
8. Vervaat $W$. A relation between Brownian bridge and Brownian excursion. - Ann. Probab., 1979, v. 7, № 1, p. 143-149.

9. Biane Ph. Relations entre pont et excursion du mouvement brownien réel. - Ann. Inst. H. Poincaré, 1986, v. 22, № 1, p. 1-7.

\title{
ОДНА МОДЕЛЬ ПРОНИКНОВЕНИЯ ГАЗА В ПОРИСТУЮ СРЕДУ
}

\begin{abstract}
В работе изучается одномерная модель проникновения газа в пористую среду. Размеры пор и прочности перегородок предполагаются независимыми и одинаково распределенными случайными величинами. В предположениях конечности дисперсии размера пор, ограниченности прочности перегородок и степенного поведения ее функции распределения доказана предельная теорема для числа пробитых перегородок.
\end{abstract}

Ключевые слова и фразы: процесс восстановления, момент выхода случайного блуждания за случайную границу, закон Вейбулла, асимптотическая независимость.

Представим себе, что газ объема $V$, находящийся под начальным давлением $P$, распространяется в пористой среде, которую в простейшем случае будем считать одномерной последовательностью ячеек, имеющих объемы $V_{i}$ и разделенных перегородками прочности $X_{i}, i=1,2, \ldots$.

Положим $T=P V$. После проникновения газа в первую пору его давление падает до величины $P_{1}$, которая находится из уравнения $P_{1}\left(V+V_{1}\right)=P V=T$, т.е. $P_{1}=T /\left(V+V_{1}\right)$. Если давление $P_{1}$ превышает прочность $X_{1}$ перегородки, разделяющей первую и вторую ячейки, то она разрушается, и газ заполняет объем $V+V_{1}+V_{2}$ (при $P_{1}=X_{1}$ будем считать, что перегородка тоже разрушается). Этот процесс продолжается до момента $N_{T}$, когда впервые оказывается, что $P_{k}<X_{k}$, где $P_{k}=T / S_{k}$, $S_{k}=V+\sum_{i=1}^{k} V_{i}, S_{0}=V$. Подобную модель для одинаковых неслучайных объемов $V_{i}$ рассматривал Д. А. Онищенко [1]. Предполагается, что выполняются следующие условия.

A1. Величина $V$ неслучайна, $V_{1}, V_{2}, \ldots$ - независимые одинаково распределенные положительные случайные величины с функцией распределения $G(x)=\mathbf{P}\left(V_{1} \leqslant x\right)$, конечным математическим ожиданием $\mu=\mathbf{M} V_{1}$ и дисперсией $\sigma^{2}=\mathbf{D} V_{1}>0$.

A2. $X_{1}, X_{2}, \ldots$ - независимые одинаково распределенные положительные случайные величины с функцией распределения $F(x)=\mathbf{P}\left(X_{1} \leqslant x\right)$ такой, что $F(c)=1$ при некотором $c>0,1-F(x) \sim b(c-x)^{\alpha}$ при $x \rightarrow c-$ и некоторых $b>0, \alpha>0$.

А3. Последовательности $\left\{V_{i}\right\}$ и $\left\{X_{i}\right\}$ независимы.

Заметим, что

$$
\begin{aligned}
\mathbf{P}\left(N_{T}>m\right) & =\mathbf{P}\left(P_{1} \geqslant X_{1}, \ldots, P_{m} \geqslant X_{m}\right)=\mathbf{P}\left(X_{1} S_{1} \leqslant T, \ldots, X_{m} S_{m} \leqslant T\right) \\
& =\mathbf{P}\left(\widehat{X}_{1} \widehat{S}_{1} \leqslant \widehat{T}, \ldots, \widehat{X}_{m} \widehat{S}_{m} \leqslant \widehat{T}\right),
\end{aligned}
$$

где $\widehat{V}_{i}=V_{i} / \mu, \widehat{X}_{i}=X_{i} / c, \widehat{T}=T /(\mu c)$. Поэтому, не ограничивая общности, в дальнейшем будем считать, что $\mu=c=1$.

* Московский государственный университет им. М.В.Ломоносова, механикоматематический факультет, Ленинские горы, 119991 Москва, Россия; е-таil: lagutinmb@mail.ru 
Теорема 1. При сделанньх предположениях и $T \rightarrow \infty$ справедливы следуюшие утверждения:

1) при $\alpha<1$ распределения величин $\left(N_{T}-T\right) T^{-1 / 2}$ сходятся $\kappa$ нормальному закону $\mathscr{N}\left(0, \sigma^{2}\right)$;

2) при $\alpha>1$ распределения величин $\left(N_{T}-T\right) T^{-\alpha /(\alpha+1)}$ сходятся $\kappa$ закону Вейбулла с функиией распределения

$$
W_{\alpha}(x)=1-e^{-b x^{\alpha+1} /(\alpha+1)}, \quad x \geqslant 0
$$

3) при $\alpha=1$ распределения величин $\left(N_{T}-T\right) T^{-1 / 2}$ сходятся $\kappa$ свертке нормального закона $\mathscr{N}\left(0, \sigma^{2}\right)$ и вейбулловского закона (1) с $\alpha=1$, а предельная функиия распределения принимает вид

$$
\Phi\left(\frac{x}{\sigma}\right)-\gamma \Phi\left(\frac{\gamma x}{\sigma}\right) e^{-b \gamma^{2} x^{2} / 2}
$$

где $\Phi(x)$ - функиия распределения закона $\mathscr{N}(0,1), \gamma=\sigma^{-1} / \sqrt{b+\sigma^{-2}}$.

Д о к а з а т е ль с т в о. Случайный момент $N_{T}=\min \left\{i \geqslant 1: S_{i}>T X_{i}^{-1}\right\}$ представляет собой момент выхода случайного блуждания $\left\{S_{i}\right\}$ за случайную границу $\left\{T X_{i}^{-1}\right\}$. Учитывая, что $X_{i}^{-1} \geqslant 1$, т.е. $T X_{i}^{-1} \geqslant T$, введем момент

$$
\tau_{T}=\min \left\{i \geqslant 1: S_{i}>T\right\} .
$$

Если величина перескока $\Delta_{T}=S_{\tau_{T}}-T \geqslant 0$ уровня $T$ превышает $T X_{\tau_{T}}^{-1}-T$, то $\tau_{T}=N_{T}$. В противном случае $N_{T}>\tau_{T}$. Обозначим ради краткости $V_{i}^{\prime}=V_{\tau_{T}+i}$, $Y_{i}=X_{\tau_{T}+i}^{-1}-1$. Положим

$$
\nu_{T}=\min \left\{k \geqslant 0: \Delta_{T}+\sum_{i=1}^{k} V_{i}^{\prime}>T Y_{k}\right\}=N_{T}-\tau_{T} .
$$

(Здесь и далее сумму $\sum_{i=1}^{k}$ полагаем равной 0 при $k=0$.)

Хорошо известно (см. [2, глава $9, \S 2]$ ), что случайные величины $\tau_{T}, V_{1}, \ldots, V_{\tau_{T}}$ и $\sigma$-алгебра, порожденная последовательностью $V_{1}^{\prime}, V_{2}^{\prime}, \ldots$, независимы, причем $V_{1}^{\prime}, V_{2}^{\prime}, \ldots$ - независимые одинаково распределенные случайные величины с функцией распределения $G(x)$. Таким образом, перенося начало координат в точку $\left(\tau_{T}, T\right)$, получим случайное блуждание, выходящее из точки с координатами $\left(0, \Delta_{T}\right)$.

С учетом введенных обозначений имеем представление

$$
\frac{N_{T}-T}{\sqrt{T}}=\frac{\tau_{T}-T}{\sqrt{T}}+\frac{\nu_{T}}{\sqrt{T}}
$$

В силу центральной предельной теоремы для процесса восстановления распределение первого слагаемого в правой части (3) сходится к закону $\mathscr{N}\left(0, \sigma^{2}\right)$ (см. [2, глава $9, \S 2$, лемма 1]).

Ниже мы докажем (см. лемму 2), что распределения величин $\nu_{T} T^{-\alpha /(\alpha+1)}$ сходятся к закону Вейбулла с функцией распределения $W_{\alpha}(x)$ из $(1)$.

Если $\alpha<1$, то $\alpha /(\alpha+1)<1 / 2$. Поэтому $\nu_{T} / \sqrt{T} \stackrel{\mathbf{P}}{\rightarrow} 0$. Отсюда и из представления (3) вытекает (лемма Слуцкого) утверждение 1) теоремы.

Если $\alpha>1$, то $\alpha /(\alpha+1)>1 / 2$. Поэтому из асимптотической нормальности $\tau_{T}$ следует, что $\left(\tau_{T}-T\right) / T^{\alpha /(\alpha+1)} \stackrel{P}{\rightarrow} 0$. Отсюда, заменяя знаменатель в $(3)$ на $T^{\alpha /(\alpha+1)}$, получаем утверждение 2) теоремы.

Наконец, в случае $\alpha=1$ воспользуемся тем, что слагаемые в правой части (3) асимптотически независимы (см. лемму 3 ). Тогда из асимптотической нормальности $\tau_{T}$ и асимптотической вейбулловости $\nu_{T}$ следует, что предельный закон для $\left(N_{T}-\right.$ $T) / \sqrt{T}$ является сверткой этих распределений (см., например, лемму 2.9.1 из [3]). Нетрудно убедиться, что функция распределения свертки выражается формулой (2). 
Перейдем к доказательству асимптотической вейбулловости $\nu_{T}$.

Известно, что при выполнении условия А1 распределение перескока $\Delta_{T}$ сходится к невырожденному закону при $T \rightarrow \infty$ (см. [2, глава $9, \S 2])$. Поэтому перескок оказывается пренебрежимо малым относительно масштабного множителя $T^{\alpha /(\alpha+1)}$. Покажем, что он не влияет на асимптотику момента $\nu_{T}$. Для этого заменим случайную величину $\Delta_{T}$ на число $c_{T} \geqslant 0$, где $c_{T} \rightarrow \infty$ при $T \rightarrow \infty$, так что $\mathbf{P}\left(\Delta_{T} \leqslant c_{T}\right) \rightarrow 1$, и введем $\widetilde{S}_{k}=c_{T}+\sum_{i=1}^{k} V_{i}^{\prime}, \widetilde{\nu}_{T}=\min \left\{i \geqslant 0: \widetilde{S}_{i}>T Y_{i}\right\}$

Лемма 1. Если $c_{T}=o\left(T^{\alpha /(\alpha+1)}\right), \operatorname{mo} \mathbf{P}\left(\widetilde{\nu}_{T} T^{-\alpha /(\alpha+1)} \leqslant x\right) \rightarrow W_{\alpha}(x)$ nрu $T \rightarrow \infty$.

Д о к а з а т е л ь с т в о. Для $x>0$ положим $m=\left[x T^{\alpha /(\alpha+1)}\right]$ (где $[\cdot]$ 一 целая часть числа) и $H(x)=\mathbf{P}\left(Y_{i}<x\right)$. Тогда

$$
\begin{aligned}
\mathbf{P}\left(\widetilde{\nu}_{T}>m\right) & =\mathbf{P}\left(\widetilde{S}_{i} \leqslant T Y_{i}, i=0, \ldots, m\right) \\
& =\int \cdots \int \prod_{i=0}^{m} I_{\left\{\tilde{s}_{i} \leqslant T y_{i}\right\}} d H\left(y_{0}\right) \cdots d H\left(y_{m}\right) d G\left(v_{1}\right) \cdots d G\left(v_{m}\right) \\
& =\int \cdots \int \prod_{i=0}^{m} \mathbf{P}\left(Y_{i} \geqslant \frac{\widetilde{s}_{i}}{T}\right) d G\left(v_{1}\right) \cdots d G\left(v_{m}\right)=\mathbf{E}\left(\prod_{i=0}^{m}\left(1-H\left(\frac{\widetilde{S}_{i}}{T}\right)\right)\right) .
\end{aligned}
$$

Далее мы покажем, что при $T \rightarrow \infty$

$$
\prod_{i=0}^{m}\left(1-H\left(\frac{\widetilde{S}_{i}}{T}\right)\right) \rightarrow e^{-b x^{\alpha+1} /(\alpha+1)} \quad \text { (п.н.). }
$$

Поскольку сомножители в произведении не превосходят 1 , применяя теорему Лебега, получаем утверждение леммы. Итак, осталось установить сходимость (4).

Легко проверить, что условие А2 влечет асимптотику $H(x) \sim b x^{\alpha}$ при $x \rightarrow 0+$. Отсюда имеем $-\ln (1-H(x)) \sim b x^{\alpha}$ при $x \rightarrow 0+$. Теперь покажем, что $\widetilde{S}_{i} / T \leqslant$ $\widetilde{S}_{m} / T \rightarrow 0$ (п.н.). Действительно,

$$
\frac{\widetilde{S}_{m}}{T}=\frac{c_{T}}{T}+\frac{m}{T} \frac{V_{1}^{\prime}+\cdots+V_{m}^{\prime}}{m} .
$$

Ввиду условия леммы имеем $c_{T} / T \rightarrow 0$. В свою очередь, $m / T \rightarrow 0,\left(V_{1}^{\prime}+\cdots+V_{m}^{\prime}\right) / m \rightarrow$ 1 (п.н.) в силу усиленного закона больших чисел (УЗБЧ). Следовательно,

$$
-\ln \left(1-H\left(\frac{\widetilde{S}_{i}}{T}\right)\right)=\left(\frac{\widetilde{S}_{i}}{T}\right)^{\alpha}\left(b+\xi_{i}\right)
$$

где $\xi_{i}=o\left(\widetilde{S}_{i} / T\right) \rightarrow 0$ (п.н.) при $T \rightarrow \infty$ равномерно по $0 \leqslant i \leqslant m$.

Суммируя, получаем

$$
-\sum_{i=0}^{m} \ln \left(1-H\left(\frac{\widetilde{S}_{i}}{T}\right)\right)=\frac{b}{T^{\alpha}} \sum_{i=0}^{m} \widetilde{S}_{i}^{\alpha}+\frac{1}{T^{\alpha}} \sum_{i=0}^{m} \widetilde{S}_{i}^{\alpha} \xi_{i}=A_{1}+A_{2} .
$$

Докажем, что $A_{1} \rightarrow b x^{\alpha+1} /(\alpha+1)$ (п.н.) и $A_{2} \rightarrow 0$ (п.н.) при $T \rightarrow \infty$. Во-первых, имеем

$$
\frac{1}{T^{\alpha}} \sum_{i=1}^{m} i^{\alpha}=\frac{m^{\alpha+1}}{T^{\alpha}} \sum_{i=1}^{m}\left(\frac{i}{m}\right)^{\alpha} \frac{1}{m} \sim \frac{m^{\alpha+1}}{T^{\alpha}} \int_{0}^{1} y^{\alpha} d y=\frac{m^{\alpha+1}}{T^{\alpha}} \frac{1}{\alpha+1} \rightarrow \frac{x^{\alpha+1}}{\alpha+1} .
$$

Далее воспользуемся леммой Тёплица из $\left[4, \S 3\right.$, лемма 1]: если $\left\{a_{n}\right\}-$ последовательность неотрицательных чисел, $b_{n}=\sum_{i=1}^{n} a_{i}, b_{n} \rightarrow \infty$ и $z_{n} \rightarrow z$ при $n \rightarrow \infty$, то $\sum_{i=1}^{n} a_{i} z_{i} \sim z b_{n}$

Возьмем $a_{m}=m^{\alpha}$ и $z_{m}=\left(\widetilde{S}_{m} / m\right)^{\alpha}$. Тогда $z_{m} \rightarrow 1$ (п.н.) при $T \rightarrow \infty$. Действительно,

$$
\frac{\widetilde{S}_{m}}{m}=\frac{c_{T}}{m}+\frac{V_{1}^{\prime}+\cdots+V_{m}^{\prime}}{m} .
$$


Ввиду условия леммы имеем $c_{T} / m \rightarrow 0,\left(V_{1}^{\prime}+\cdots+V_{m}^{\prime}\right) / m \rightarrow 1$ (п.н.) в силу УЗБЧ. Следовательно,

$$
\frac{1}{T^{\alpha}} \sum_{i=1}^{m} \widetilde{S}_{i}^{\alpha}=\frac{1}{T^{\alpha}} \sum_{i=1}^{m} i^{\alpha}\left(\frac{\widetilde{S}_{i}}{i}\right)^{\alpha} \rightarrow \frac{x^{\alpha+1}}{\alpha+1} \quad \text { (п.н.). }
$$

Слагаемое в $A_{1}$ при $i=0$ можно не учитывать, так как $\widetilde{S}_{0}=c_{T}=o(T)$ и $c_{T}^{\alpha} / T^{\alpha} \rightarrow 0$.

Во-вторых, опираясь на полученный результат и равномерную малость $\xi_{i}$ (п.н.), выводим, что $A_{2} \rightarrow 0$ (п.н.) при $T \rightarrow \infty$. Лемма 1 доказана.

Лемма 2. $\mathbf{P}\left(\nu_{T} T^{-\alpha /(\alpha+1)} \leqslant x\right) \rightarrow W_{\alpha}(x)$ nрu $T \rightarrow \infty$.

Д ок аз а т е ль с т в о. Заметим, что если $\mathbf{P}\left(A_{T}\right) \rightarrow p, \mathbf{P}\left(B_{T}\right) \rightarrow 1$, то $\mathbf{P}\left(A_{T} B_{T}\right) \rightarrow p$. Возьмем в качестве $B_{T}$ события $\left\{\Delta_{T} \leqslant c_{T}\right\}$, где $c_{T} \geqslant 0$. Как и прежде, для $x>0$ положим $m=\left[x T^{\alpha /(\alpha+1)}\right]$. Тогда

$$
\begin{aligned}
\mathbf{P}\left(\nu_{T}>m\right) & =\mathbf{P}\left(\Delta_{T} \leqslant T Y_{0}, \ldots, \Delta_{T}+V_{1}^{\prime}+\cdots+V_{m}^{\prime} \leqslant T Y_{m}\right) \\
& \geqslant \mathbf{P}\left(\Delta_{T} \leqslant T Y_{0}, \ldots, \Delta_{T}+V_{1}^{\prime}+\cdots+V_{m}^{\prime} \leqslant T Y_{m}, \Delta_{T} \leqslant c_{T}\right) \\
& \geqslant \mathbf{P}\left(c_{T} \leqslant T Y_{0}, \ldots, c_{T}+V_{1}^{\prime}+\cdots+V_{m}^{\prime} \leqslant T Y_{m}, \Delta_{T} \leqslant c_{T}\right) .
\end{aligned}
$$

Пусть $c_{T} \rightarrow \infty, c_{T}=o\left(T^{\alpha /(\alpha+1)}\right)$ при $T \rightarrow \infty$. Тогда $\mathbf{P}\left(\Delta_{T} \leqslant c_{T}\right) \rightarrow 1$, и из леммы 1 и вышеприведенного замечания следует неравенство

$$
\underline{\lim } \mathbf{P}\left(\nu_{T}>m\right) \geqslant e^{-b x^{\alpha+1} /(\alpha+1)}
$$

Для получения оценки сверху заменим перескок $\Delta_{T}$ на 0:

$$
\mathbf{P}\left(\nu_{T}>m\right) \leqslant \mathbf{P}\left(V_{1}^{\prime} \leqslant T Y_{1}, \ldots, V_{1}^{\prime}+\cdots+V_{m}^{\prime} \leqslant T Y_{m}\right) .
$$

Применяя лемму 1 с $c_{T}=0$ к правой части, выводим неравенство

$$
\varlimsup \mathbf{P}\left(\nu_{T}>m\right) \leqslant e^{-b x^{\alpha+1} /(\alpha+1)} .
$$

Лемма 2 доказана.

Наконец, рассмотрим случай $\alpha=1$.

Лемма 3. Для произвольных действительньх $x \in \mathbf{R}$ и $y>0$ при $T \rightarrow \infty$

$$
\mathbf{P}\left(\tau_{T} \leqslant T+x \sqrt{T}, \nu_{T}>y \sqrt{T}\right) \rightarrow \Phi\left(\frac{x}{\sigma}\right) e^{-b y^{2} / 2} .
$$

Д о к а з а т е ль с т в о. Применим ту же схему рассуждения, что и в лемме 2. Положим $m=[y \sqrt{T}], D_{T}=\left\{\tau_{T} \leqslant T+x \sqrt{T}\right\}$. Тогда

$$
\begin{aligned}
\mathbf{P}\left(\tau_{T}\right. & \left.\leqslant T+x \sqrt{T}, \nu_{T}>y \sqrt{T}\right)= \\
& =\mathbf{P}\left(D_{T}, \Delta_{T} \leqslant T Y_{0}, \ldots, \Delta_{T}+V_{1}^{\prime}+\cdots+V_{m}^{\prime} \leqslant T Y_{m}\right) \\
& \geqslant \mathbf{P}\left(D_{T}, \Delta_{T} \leqslant T Y_{0}, \ldots, \Delta_{T}+V_{1}^{\prime}+\cdots+V_{m}^{\prime} \leqslant T Y_{m}, \Delta_{T} \leqslant c_{T}\right) \\
& \geqslant \mathbf{P}\left(D_{T}, c_{T} \leqslant T Y_{0}, \ldots, c_{T}+V_{1}^{\prime}+\cdots+V_{m}^{\prime} \leqslant T Y_{m}, \Delta_{T} \leqslant c_{T}\right) .
\end{aligned}
$$

Рассмотрим события

$$
A_{T}=\left\{D_{T}, c_{T} \leqslant T Y_{0}, \ldots, c_{T}+V_{1}^{\prime}+\cdots+V_{m}^{\prime} \leqslant T Y_{m}\right\}, \quad B_{T}=\left\{\Delta_{T} \leqslant c_{T}\right\} .
$$

Используя независимость $\tau_{T}$ от $\left\{V_{i}^{\prime}\right\}$ и $\left\{Y_{i}\right\}$, получаем:

$$
\mathbf{P}\left(A_{T}\right)=\mathbf{P}\left(D_{T}\right) \mathbf{P}\left(c_{T} \leqslant T Y_{0}, \ldots, c_{T}+V_{1}^{\prime}+\cdots+V_{m}^{\prime} \leqslant T Y_{m}\right) .
$$

В силу центральной предельной теоремы $\mathbf{P}\left(D_{T}\right) \rightarrow \Phi(x / \sigma)$ при $T \rightarrow \infty$. Согласно лемме 1 , второй сомножитель при $c_{T} \rightarrow \infty, c_{T}=o\left(T^{1 / 2}\right)$ стремится к $e^{-b y^{2} / 2}$. Поэтому $\mathbf{P}\left(A_{T}\right) \rightarrow \Phi(x / \sigma) e^{-b y^{2} / 2}$. Таким образом,

$$
\underline{\varliminf} \mathbf{P}\left(\tau_{T} \leqslant T+x \sqrt{T}, \nu_{T}>y \sqrt{T}\right) \geqslant \Phi\left(\frac{x}{\sigma}\right) e^{-b y^{2} / 2} .
$$


Для получения оценки сверху заменим $\Delta_{T}$ на 0 :

$$
\begin{aligned}
\mathbf{P}\left(\tau_{T} \leqslant T+x \sqrt{T}, \nu_{T}>y \sqrt{T}\right) & \leqslant \mathbf{P}\left(D_{T}, V_{1}^{\prime} \leqslant T Y_{1}, \ldots, V_{1}^{\prime}+\cdots+V_{m}^{\prime} \leqslant T Y_{m}\right) \\
& =\mathbf{P}\left(D_{T}\right) \mathbf{P}\left(V_{1}^{\prime} \leqslant T Y_{1}, \ldots, V_{1}^{\prime}+\cdots+V_{m}^{\prime} \leqslant T Y_{m}\right) .
\end{aligned}
$$

Применяя к сомножителям в правой части центральную предельную теорему и лемму 1 с $c_{T}=0$, выводим неравенство

$$
\varlimsup \mathbf{P}\left(\tau_{T} \leqslant T+x \sqrt{T}, \nu_{T}>y \sqrt{T}\right) \leqslant \Phi\left(\frac{x}{\sigma}\right) e^{-b y^{2} / 2} .
$$

Объединяя (6) и (7), приходим к (5). Лемма 3 , а вместе с ней и теорема доказаны.

\section{СПИСОК ЛИТЕРАТУРЫ}

1. Онищенко Д. А. Вероятностная модель пробоя крупноячеистой пены в пористой среде. Препринт №681. М.: Ин-т проблем механики РАН, 2001, 31 с.

2. Боровков A. А. Теория вероятностей. М.: Наука, 1986, 432 с.

3. Галамбош Я. Асимптотическая теория экстремальных порядковых статистик. М.: Наука, 1984, 304 с.

4. Ширяев А.Н. Вероятность. М.: Наука, 1989, 640 с.

Поступила в редакцию

29.IX.2011

(c) 2012 г.

DINEV T.* MATTNER L.* THE ASYMPTOTIC BERRY-ESSEEN CONSTANT
FOR INTERVALS

Показано, что асимптотическая константа в неравенстве БерриЭссена для интервальных вероятностей равна $\sqrt{2 / \pi}$.

Ключевые слова и фразы: неравенство Берри-Эссена, интервальные вероятности, асимптотическая константа.

1. Introduction. Let $\mathbf{R}$ denote the real numbers, $\mathbf{Z}$ the integers, and $\mathbf{N}$ the integers $\geqslant 1$. Let $\operatorname{Prob}(\mathbf{R})$ be the set of all (probability) laws on $\mathbf{R}, \delta_{x}$ the unit mass at $x \in \mathbf{R}$, and $\mathrm{N}_{0,1}$ the standard normal law with distribution function $\Phi$ and density $\varphi:=\Phi^{\prime}$.

For $P \in \operatorname{Prob}(\mathbf{R})$ and $s \in\left[1, \infty\left[\right.\right.$, let $\mu(P):=\int x P(\mathrm{~d} x)$ and $\beta_{s}(P):=\int \mid x-$ $\left.\mu(P)\right|^{s} P(\mathrm{~d} x)$ if $\int|x| P(\mathrm{~d} x)<\infty, \beta_{s}(P):=\infty$ otherwise, $\sigma^{2}(P):=\beta_{2}(P)$, and $\alpha(P):=$ $\int(x-\mu(P))^{3} P(\mathrm{~d} x)$ if $\beta_{3}(P)<\infty$. If $P$ is a lattice law, let $h(P):=\sup \bigcup_{a \in \mathbf{R}}\{\eta \in$ ] $0, \infty[: P(a+\eta \mathbf{Z})=1\}$, otherwise set $h(P):=0$. Let us put

$$
\mathscr{P}_{s}:=\left\{P \in \operatorname{Prob}\left(\mathbf{R}: 0<\beta_{s}(P)<\infty\right\} .\right.
$$

In the following, we shall consider sequences $\left(X_{k}\right)_{k \in \mathbf{N}}$ of independent and identically distributed real-valued random variables. $P$ will then stand for the law of $X_{1}$ and shall, for the sake of brevity, usually be omitted in the quantities $\mu, \sigma^{2}, \alpha, \beta_{s}$, and $h$ just defined. Let $P_{n} \in \operatorname{Prob}(\mathbf{R})$ be given by $P_{n}(A):=P^{* n}(\sigma \sqrt{n} A+n \mu)$ and set $\left.\left.F_{n}(x):=P_{n}(]-\infty, x\right]\right)$ for

* Universität Trier, Fachbereich IV — Mathematik, 54296 Trier, Germany; e-mail: dinev@uni-trier.de, mattner@uni-trier.de

1) This research was partially supported by DFG grant MA 1386/3-1. 